patients, however their ligands, CXCL8 and CXCL6, were lost from the territorial matrix of chondrocytes. Blockade of CXCR1/2 signalling at receptor level in chondrocytes resulted in a significantly reduced extracellular matrix sulphated glycosaminoglycan content of micromass cultured chondrocytes and a significantly reduced expression of the chondrocyte differentiation markers COL2A1, Aggrecan and SOX9. CXCR2 and CXCL5 were expressed in unchallenged wild type mouse articular cartilage. CXCR2 $\%$ mice subjected to the DMM model and analysed 8 weeks following surgery developed a significantly more severe osteoarthritis phenotype than wild type controls.

Conclusions Our findings indicate that CXCR1/2 signalling is required for the maintenance of phenotypic stability of articular chondrocytes. We show that mouse CXCR2 signalling is required for articular cartilage homeostasis and is chondroprotective during conditions of challenge in vivo.

\section{A8.18 VISFATIN/PBEF IN BONE REMODELLING OF RHEUMATOID ARTHRITIS}

doi:10.1136/annrheumdis-2013-203222.18

'G Krumbholz, 'S Junker, ${ }^{2} \mathrm{~A}$ Lehr, ${ }^{3} \mathrm{M}$ Rickert, ${ }^{4} \mathrm{G}$ Schett, 'U Lange, ${ }^{2} \mathrm{~S}$ Rehart, 'U Müller-Ladner, 'E Neumann. 'Dept Internal Medicine and Rheumatology, JustusLiebig-University Gießen, Kerckhoff-Klinik Bad Nauheim, Germany; ${ }^{2}$ Dept Orthopedics and Trauma Surgery, Markus-Hospital, Frankfurt, Germany; ${ }^{3}$ Dept Orthopedics and Orthopedic Surgery, University Hospital Gießen and Marburg, Gießen, Germany; ${ }^{4}$ Medical Clinic 3: Rheumatology and Immunology, Erlangen, Germany

Objectives Rheumatoid arthritis (RA) is associated with increased production of the adipocytokine visfatin in synovial fluid and tissue of RA patients. Visfatin promotes the synthesis of pro-inflammatory and matrix-degrading effector molecules in RA synovial fibroblasts. Moreover, an immunohistochemical analysis of RA bone tissue showed a co-localisation of visfatin with key cells of bone remodelling (osteoblasts, osteoclasts) but the role of this adipokine in processes of bone remodelling in RA is unclear. In this study, we focussed on visfatin and its influence on RA osteoblast and osteoclast activity and differentiation as well as on its immunomodulatory properties.

Methods Human osteoblasts and osteoclasts were isolated from bone tissue and blood samples of RA patients and stimulated with visfatin. Visfatin-mediated effects on osteoblasts and osteoclasts were analysed on the transcriptional and translational level using realtime polymerase chain reaction and immunoassays. Additionally, effects of visfatin on matrix-production of osteoblasts as well as differentiation and resorption activity of osteoclasts were examined by Alizarin-Red S-, TRAP- and von Kossa-staining.

Results Stimulation with visfatin induced the secretion of pro-inflammatory cytokines (e.g. IL-6: 5-fold increase; IL-8: up to 100 -fold) in RA osteoblasts. Additionally, quantitative realtime PCR showed several genes being differentially expressed in osteoblasts after stimulation with visfatin (e.g. alkaline phosphatase, OPG, Osterix). In contrast, osteoclasts only weakly respond to visfatin. A regulation on translational level was observed with regard to the production of the cytokines IL- 6 and IL-8, showing a moderate increase.

Conclusions The results of the present study indicate that visfatin influences the activity as well as the differentiation of human osteoblasts in RA by modulating the expression of genes being involved in matrix production and osteoblast phenotype development. These results support the idea of visfatin affecting bone metabolism in RA. Furthermore, the finding of cytokine-induction in osteoblasts and osteoclasts in RA confirm the pro-inflammatory potential of visfatin in RA.

Acknowledgement Funded by the German Research Society (SPP1468, IMMUNOBONE, NE1174/6-1).

\section{Novel therapeutic targets}

\section{A9.1 AVB3 INTEGRIN INHIBITION WITH CILENGITIDE BOTH PREVENTS AND TREATS COLLAGEN INDUCED ARTHRITIS}

doi:10.1136/annrheumdis-2013-203223.1

'Despoina Sykoutri, ${ }^{2}$ Nisha Geetha, 'Silvia Hayer, ${ }^{1}$ Peter Mandl, ${ }^{1}$ Josef S Smolen, ${ }^{2}$ Gerald Prager, ${ }^{1}$ Kurt Redlich. 'Department of Rheumatology; ${ }^{2}$ Department of Oncology, Medical University of Vienna, Austria

Background Rheumatoid arthritis (RA) is characterised by synovial inflammation and osteoclast $(\mathrm{OC})$ mediated bone erosions. AlphaVbeta3 ( $\alpha v \beta 3)$ integrin is highly expressed in OCs. Av $\beta 3$ blocking antibodies reduce bone resorption and mice lacking $\beta 3$ are osteopetrotic.

Objectives Efficacy testing of the $\alpha v \beta 3$ inhibitor cilengitide, a synthetic Arginine-Glycine-Asparagine amino acid peptide (RGD peptide), on osteoclastogenesis and the collagen induced arthritis (CIA) model for human RA.

Materials and Methods In vitro mouse bone marrow-derived cells (BMCs) were differentiated into tartrate resistant acid phosphatase positive (TRAP + ) mononuclear OC precursor cells (pre$\mathrm{OCs}$ ) and TRAP+ multinucleated mature OCs with macrophagecolony stimulating factor (M-CSF) and receptor activator of nuclear factor kappaB ligand (RANKL). Cilengitide, was added in increasing concentrations $(2 \mathrm{nM}$ to $20 \mu \mathrm{M})$ to the culture. These osteoclastogenesis assays were performed on plates coated with RGD containing matrixes osteopontin, fibronectin and fibrinogen but also on Poly-D-lysine to assess $\alpha v \beta 3$ independent adhesion. In vivo CIA was induced in 6-8 week old male DBA/1 mice by immunisation with bovine type II collagen at day 1 , followed by boosting at day 21 . For CIA prevention mice received subcutaneously (s.c.) $1.5 \mathrm{mg} / \mathrm{kg}$ cilengitide $(n=15)$ or placebo $(n=15), 5$ days per week, 1 day prior to CIA induction until day 53. For CIA treatment, mice with arthritis were randomised and received $1.5 \mathrm{mg} / \mathrm{kg}$ (low dose, $\mathrm{n}=19$ ) or $75 \mathrm{mg} / \mathrm{kg}$ (high dose, $\mathrm{n}=7$ ) cilengitide or placebo $(\mathrm{n}=21$ ), until day 59. Preventive and treatment effects were evaluated by assessing paw thickness and grip strength.

Results In vitro increasing concentrations of cilengitide (IC50: $250 \mathrm{nM}$ ) dose-dependently reduced pre-OCs on all coatings, indicating early inhibition at the pre-OC proliferation stage. OCs were reduced above $200 \mathrm{nM}$, followed by complete disappearance above $2 \mu \mathrm{M}$. At $200 \mathrm{nM}$ an intriguing morphological difference with reduction in $O C$ size suggested that cilengitide may disrupt spreading and fusion capacity at the early pre-OC stage. In vivo CIA prevention with cilengitide effectively reduced incidence $(92.8 \%$ versus $40 \%$ ) and severity of arthritis as evidenced by reduction of clinical disease activity scores. Low and high dose cilengitide effectively inhibited progression of established arthritis.

Conclusions Osteoclastogenesis requires intact $\alpha v \beta 3$ integrin function. Systemic $\alpha v \beta 3$ integrin inhibition with cilengitide potently prevents and treats experimental CIA. Cilengitide may be a novel therapeutic target in RA.

\section{A9.2 THE PHOSPHOINOSITIDE 3-KINASE PATHWAY REGULATES FIBROBLAST-LIKE SYNOVIOCYTES INVASION}

doi:10.1136/annrheumdis-2013-203223.2

Beatrix Bartok, Gary S Firestein. UC San Diego School of Medicine, La Jolla, CA

Background/Objectives Cartilage destruction mediated by invasive fibroblast-like synoviocytes (FLS) plays a central role in pathogenesis of RA. Increased cell migration and degradation of extracellular matrix are fundamental to these processes. The Class I phosphoinositide 3-kinases (PI3K) control cell survival, proliferation and migration, which might be involved with cartilage damage in 
RA. PI3Kdelta isoform expression was recently identified as a key regulator of FLS growth and survival, suggesting that it could contribute to synoviocyte aggressive behaviour. Therefore, we assessed the role of PI3Kdelta in synoviocyte invasion and matrix degradation using isoform selective PI3K inhibitors.

Methods FLS were cultured in Matrigel coated transwells. PI3K inhibitors or vehicle were added to the upper chamber and PDGF was used as a chemoattractant in the lower chamber. The invading cells were quantified by staining the philtres with $1 \%$ crystal violet. F-actin was visualised with Rhodamin phalloidin and analysed with fluorescent microscopy. Rac1 activation was measured using PAK1/ PBD GST pull down and quantified by Western blot analysis. PI3K inhibitors: pan (GDC-0941), PI3Kalpha (A66), PI3Kbeta (TGX-221), PI3Kgamma (AS-252424), PI3Kdelta (INK007 and CAL-101) and PI3K-delta/gamma (IPI-145).

Results PDGF-directed invasion was completely inhibited by the pan PI3K inhibitor ( $1 \mathrm{uM})$. To define the role of the individual isoforms, we tested the effect of the isoform selective PI3K inhibitors. PI3Kdelta inhibition (INK007) significantly decreased the number of invading cells, with $60 \pm 5 \%$ inhibition at $1 \mathrm{uM}(\mathrm{p}<0.04)$. Similar results were observed with two other inhibitors with distinct chemical structures (CAL-101 and INK055). The PI3Kalpha inhibitor decreased invasion by $40 \pm 5 \%$ while PI3Kbeta and PI3Kgamma inhibitors had no effect. Phalloidin staining was then used to visualise FLS actin rearrangement in response to PDGF with or without PI3K inhibitors. PI3Kdelta inhibition by INK007, CAL-101 and IPI-145 decreased lamellipodia formation by $50 \pm 6 \%(p<0.05)$. Similar inhibition was seen with the pan PI3K inhibitor, while the selective inhibitors of PI3Kalpha, PI3Kbeta or PI3Kgamma had no effect. We then hypothesised that PI3Kdelta might modulate activation of Rho GTPases in synoviocytes, which regulate actin organisation. PI3Kdelta inhibition with INK007 had no effect on baseline Rac1 activation but blocked activation in response to PDGF by $95 \pm 6 \%$ $(p<0.03)$. Similar findings were observed with the pan PI3K inhibitor, while PI3Kalpha inhibition had no significant effect.

Conclusions PI3Kdelta is a major regulator of FLS migration and invasion and functions by inhibiting Rac1 activation and modulating $\mathrm{F}$ actin cytoskeleton rearrangement. These observations, together with previous findings that PI3Kdelta regulates FLS growth and survival, suggest that PI3Kdelta inhibition could be chondroprotective in RA by modulating synoviocyte growth, migration and invasion.

\section{A9.3 DELETION OF mPGES-1 AFFECTS FATTY ACID COMPOSITION AND EICOSANOID PROFILES IN MICE}

doi:10.1136/annrheumdis-2013-203223.3

\begin{abstract}
1,2Helena Idborg, ${ }^{3}$ Petter Olsson, 'Patrick Leclerc, ' 'Joan Raouf, 'Per-Johan Jakobsson, 1,2Marina Korotkova. 'Rheumatology Unit, Department of Medicine, Karolinska Institutet, Sweden; ${ }^{2}$ Actar AB, Solna, Sweden; ${ }^{3}$ Department of Analytical Chemistry, Stockholm University, Sweden
\end{abstract}

Background mPGES-1 is considered an attractive alternative target for anti-inflammatory treatment with improved selectivity and safety compared to NSAIDs. Genetic deletion or pharmacological inhibition of mPGES-1 activity or expression down-regulate inflammation and pain in experimental models of arthritis. However, a detailed understanding of the molecular mechanisms and pathways affected by deletion/inhibition of mPGES-1 is essential before mPGES-1 inhibitors can safely be applied in the clinics.

Objectives To investigate the effect of mPGES-1 deletion on the levels of bioactive lipid mediators such as the eicosanoids (downstream cascade) and fatty acids (up-stream cascade).

Methods Peritoneal macrophages (PM) from wild type (WT) and knock-out (mPGES-1\%, KO) mice were induced with LPS for $16 \mathrm{~h}$. Cells were harvested for gene expression analysis and supernatants were collected for eicosanoid analysis. Gene expression profiling in
WT and $\mathrm{KO}$ macrophages was performed using the microarray analysis (Applied Biosystems). Eicosanoid profiling of approximately 30 compounds was performed using LC-MS/MS. Fatty acid composition of total lipids in spleen and brain homogenates of WT and $\mathrm{KO}$ mice were determined using GC/FID.

Results Microarray analysis revealed that genetic deletion of mPGES-1 affected expression of genes related to lipid metabolism and mainly associated with eicosanoid, fatty acid and phospholipid metabolism, (e.g., Pla1A, Ptgis, Fabp3, Cept1, Comt etc). Compared to WT, mPGES-1 deficient PM displayed a markedly attenuated increase in $\mathrm{PGE}_{2}$ production upon LPS stimulation, and exhibited significantly increased levels of $\mathrm{PGD}_{2}$ metabolites such as 15 -deoxy- $\Delta^{12,14}$ PGJ ${ }_{2}$ and 15 -deoxy- $\Delta^{12,14} \mathrm{PGD}_{2}(\mathrm{p}<0.05)$. There were significant differences in the fatty acid composition in spleen (e.g., palmitoleic acid decreased in $\mathrm{KO}, \mathrm{p}<0.05$ ) and brain (e.g., myristic acid decreased in $\mathrm{KO}, \mathrm{p}<0.05$ ) of $\mathrm{KO}$ and WT mice, suggesting that feed-back mechanisms are alternated by the deletion of m-PGES-1.

Conclusions Data reveals that mPGES-1 deficient PMs displayed shunting towards PGD2 pathway, i.e., towards anti-inflammatory metabolites, upon LPS stimulation compared to WT PMs. Moreover, mPGES-1 depletion alters the fatty acid composition of tissue lipids.

These effects of inducible $\mathrm{PGE}_{2}$ on lipid metabolism have important implications for future mPGES-1 inhibitors and deserve further investigation.

\section{A9.4 FLT3L-DEPENDENT CD103 + DC ARE CRUCIAL FOR THE INITIATION AND MAINTENANCE OF COLLAGEN-INDUCED ARTHRITIS}

doi:10.1136/annrheumdis-2013-203223.4

1,2M Inês Ramos, 1,2Saida Aarrass, ${ }^{3}$ SE Jacobsen, 'Paul P Tak, 1,2M Cristina Lebre. ${ }^{1}$ Departmtment of Clinical Immunology and Rheumatology; ${ }^{2}$ Department of Experimental Immunology, Academic Medical Center/University of Amsterdam, Amsterdam, The Netherlands; ${ }^{3}$ Stem Cell Biology Laboratory, University of Oxford, UK

Background and Objectives Dendritic cells (DCs) are a heterogeneous population of antigen-sensing and-presenting cells that play an important role for the initiation of protective and pathogenic immunity. Flt3L is a growth factor that can drive DC development from bone marrow (BM) progenitors and it is crucial for steadystate DC maintenance. Collagen-induced arthritis (CIA) induction requires dermal DC uptake, processing, migration and presentation of antigen to lymph node (LN) T cells. The objective of this study was to find the mechanism of protection in CIA observed in mice lacking Flt3L (Flt3L $\mathrm{L}^{-/}$).

Materials and Methods CIA was induced in Flt3L $\mathrm{L}^{-1}$ and WT littermates. The severity of the arthritis was assessed using an established semiquantitative scoring system (0-4). In vitro uptake and migration was assessed by using BM-DC (differentiated with GM-CSF for9days). OVA-FITCwasinjectedintradermallyin Flt3L $\mathrm{L}^{-1}$ and WT animals, after $36 \mathrm{~h}$ LNs were collected and the number of FITC positive cells was determined. Antigen presentation was studied by adoptive transfer of CFSE-labelled OT-I or OT-II T cells + OVA (i.d.) into Flt $3 \mathrm{~L}^{-/}$and WT animals. After 3 days LNs were collected and $\mathrm{T}$ cell proliferation was analysed by CFSE dilution.

Results $\mathrm{Flt} 3 \mathrm{~L}^{-/}$mice (steady-state) have severe reductions in DC populations in lymphoid and non-lymphoid organs. Flt3 $3 \mathrm{~L}^{-1-}$ mice are protected from CIA showing impaired $\mathrm{T}$ and $\mathrm{B}$ cell responses. Flt $3 \mathrm{~L}^{-1}$ mice have reduced dermal CD11b + DCs and CD103+ DCs are almost absent. In addition, the amount of DCs carrying antigen reaching the $\mathrm{LN}$ in $\mathrm{Flt} 3 \mathrm{~L}^{-1}$ mice was reduced compared with WT. In vitro and in vivo experiments using BMDCs demonstrated that Flt $3 \mathrm{~L}^{-}$BMDCs have the same capacity to uptake and migrate as WT BMDCs. Moreover, adoptive transfer of OT-I and OT-II T cells + OVA in Flt3 $\mathrm{L}^{-/}$mice resulted in a dramatic reduction of total cell 